\title{
ON RATIONAL CLASSICAL ORTHOGONAL POLYNOMIALS AND THEIR APPLICATION FOR EXPLICIT COMPUTATION OF INVERSE LAPLACE TRANSFORMS
}

\author{
MOHAMMAD MASJED-JAMEI AND MEHDI DEHGHAN
}

Received 26 June 2004 and in revised form 21 August 2004

From the main equation $\left(a x^{2}+b x+c\right) y_{n}^{\prime \prime}(x)+(d x+e) y_{n}^{\prime}(x)-n((n-1) a+d) y_{n}(x)=0$, $n \in \mathbb{Z}^{+}$, six finite and infinite classes of orthogonal polynomials can be extracted. In this work, first we have a survey on these classes, particularly on finite classes, and their corresponding rational orthogonal polynomials, which are generated by Mobius transform $x=p z^{-1}+q, p \neq 0, q \in \mathbb{R}$. Some new integral relations are also given in this section for the Jacobi, Laguerre, and Bessel orthogonal polynomials. Then we show that the rational orthogonal polynomials can be a very suitable tool to compute the inverse Laplace transform directly, with no additional calculation for finding their roots. In this way, by applying infinite and finite rational classical orthogonal polynomials, we give three basic expansions of six ones as a sample for computation of inverse Laplace transform.

\section{Introduction}

It is known that the Jacobi, Laguerre, Hermite, and Bessel classical orthogonal polynomials are special cases of the polynomial solutions of the differential equation

$$
\left(a x^{2}+b x+c\right) y_{n}^{\prime \prime}(x)+(d x+e) y_{n}^{\prime}(x)-n((n-1) a+d) y_{n}(x)=0
$$

in which $a, b, c, d$, e are real parameters and $n$ is a positive integer number. So far, extensive researches have been done on this equation [16, 24]. Bochner in 1929 [2] might be the first person who was able to classify the polynomial solutions of (1.1) in the following forms:

(i) Jacobi polynomials $\left\{P_{n}^{(\alpha, \beta)}(x)\right\}_{n=0}^{\infty}(\alpha, \beta, \alpha+\beta+1 \notin\{-1,-2, \ldots\})$;

(ii) Laguerre polynomials $\left\{L_{n}^{(\alpha)}(x)\right\}_{n=0}^{\infty}(\alpha \notin\{-1,-2, \ldots\})$;

(iii) Hermite polynomials $\left\{H_{n}(x)\right\}_{n=0}^{\infty}$;

(iv) Bessel polynomials $\left\{B_{n}^{(\alpha, \beta)}(x)\right\}_{n=0}^{\infty}(\alpha \notin\{0,-1,-2, \ldots\}$ and $\beta \neq 0)$;

(v) $\left\{x^{n}\right\}_{n=0}^{\infty}$.

The cases (i), (ii), (iii), and (iv) are infinitely orthogonal while the case (v) is not orthogonal for any weighting function. Nowadays, the first four cases are known as the classical orthogonal polynomials. But recently in $[13,14]$, we have showed that in fact six series of 
Table 1.1

\begin{tabular}{lcccccc}
\hline Kind & Name & $a$ & $b$ & $c$ & $d$ & $e$ \\
\hline (1) Infinite & $P_{n}^{(\alpha, \beta)}(x)$ & -1 & 0 & 1 & $-\alpha-\beta-2$ & $-\alpha+\beta$ \\
(2) Infinite & $L_{n}^{(\alpha)}(w x)$ & 0 & 1 & 0 & $-w$ & $\alpha+1$ \\
(3) Infinite & $H_{n}(w x+v / 2 w)$ & 0 & 0 & 1 & $-2 w^{2}$ & $-v$ \\
(4) Finite & $M_{n}^{(p, q)}((w / v) x)$ & $w$ & $v$ & 0 & $(-p+2) w$ & $(q+1) v$ \\
(5) Finite & $N_{n}^{(p)}(w x)$ & $w$ & 0 & 0 & $(-p+2) w$ & 1 \\
(6) Finite & $J_{n}^{(p, q)}(x ; a, b, c, d)$ & $a^{*}$ & $b^{*}$ & $c^{*}$ & $d^{*}$ & $e^{*}$ \\
\hline$a^{*}=a^{2}+c^{2}, b^{*}=2(a b+c d), c^{*}=b^{2}+d^{2}, d^{*}=2(1-p)\left(a^{2}+c^{2}\right)$, & & \\
$e^{*}=q(a d-b c)+2(1-p)(a b+c d)$. & & & &
\end{tabular}

classical orthogonal polynomials can be extracted from the main equation (1.1). Three series of them, as was said, are "infinitely" orthogonal for every $n$ and three other ones, as we have indicated in $[13,14]$, are "finitely" orthogonal for limited $n$.

Table 1.1 shows the values $a, b, c, d, e$ from (1.1) corresponding to each six classes.

We here mention that we have called these three finite sequences as $J_{n}^{(p, q)}(x ; a, b, c, d)$, $M_{n}^{(p, q)}(x)$, and $N_{n}^{(p)}(x)$ merely for following the alphabetical arrangement.

The first finite class $\left\{M_{n}^{(p, q)}(x)\right\}_{n=0}^{n=N}$ is an orthogonal set with respect to the weight function $W_{1}(x, p, q)=x^{q} /(1+x)^{p+q}$ on $[0, \infty)$ if and only if $q>-1$ and $p>2 N+1[13$, Section 1]. For instance, $\left\{M_{n}^{(p, q)}(x)\right\}_{n=0}^{n=100}$ is a finite orthogonal set on $[0, \infty)$ with respect to $W_{1}(x, p, q)$ if and only if $q>-1$ and $p>201$. Of course note that the finite sets of orthogonal polynomials are usually used for the discrete orthogonal polynomials rather than continuous cases. For example, the Krawtchouk polynomials [10, Section 1.10] that are defined as

$$
K_{n}(x, p, N)={ }_{2} F_{1}\left(-n,-x,-N ; \frac{1}{p}\right), \quad n=0,1,2, \ldots, N
$$

and satisfy the orthogonality relation

$$
\sum_{x=0}^{N}\left(\begin{array}{c}
N \\
x
\end{array}\right) P^{x}(1-p)^{N-x} K_{n}(x) K_{m}(x)=\frac{(-1)^{n} n !}{(-N)_{n}}\left(\frac{1-p}{p}\right)^{n} \delta_{n, m}, \quad 0<p<1,
$$

in which $(n)_{k}=\Gamma(n+k) / \Gamma(n)$ and

$$
\delta_{n, m}= \begin{cases}0 & \text { if } n \neq m \\ 1 & \text { if } n=m\end{cases}
$$

are a sample of this comment.

Moreover, the Racah polynomials [10, Section 1.2] and Dual Hahn discrete polynomials [10, Section 1.6] are two other finite discrete orthogonal polynomials that have been applied in the Askey scheme of hypergeometric orthogonal polynomials. 
But the polynomials

$$
M_{n}^{(p, q)}(x)=(-1)^{n} n ! \sum_{k=0}^{k=n}\left(\begin{array}{c}
p-(n+1) \\
k
\end{array}\right)\left(\begin{array}{l}
q+n \\
n-k
\end{array}\right)(-x)^{k} \Longleftrightarrow\left(\begin{array}{l}
a \\
k
\end{array}\right)=\frac{1}{k !} \prod_{i=0}^{k-1}(a-i)
$$

satisfy the orthogonality relation $[13$, Section 1$]$

$$
\int_{0}^{\infty} \frac{x^{q}}{(1+x)^{p+q}} M_{n}^{(p, q)}(x) M_{m}^{(p, q)}(x) d x=\frac{n !(p-n-1) !(q+n !)}{(p-2 n-1)(p+q-n-1) !} \delta_{n, m}
$$

if and only if $m, n=0,1,2, \ldots, N<(p-1) / 2, q>-1$.

For instance, the polynomial set

$$
\left\{M_{n}^{(202,0)}(x)\right\}_{n=0}^{100}=\left\{(-1)^{n} n ! \sum_{k=0}^{k=n}\left(\begin{array}{c}
201-n \\
k
\end{array}\right)\left(\begin{array}{l}
n \\
k
\end{array}\right)(-x)^{k}\right\}_{n=0}^{100}
$$

is finitely orthogonal with respect to the weight function $W_{1}(x, 202,0)=(1+x)^{-202}$ on $[0, \infty)$ and

$$
\int_{0}^{\infty}(1+x)^{-202} M_{n}^{(202,0)}(x) M_{m}^{(202,2)}(x) d x=\frac{(n !)^{2}}{201-2 n} \delta_{n, m} \Longleftrightarrow m, n \leq 100 .
$$

On the other hand, since the Jacobi polynomials have a direct relation with these polynomials, that is,

$$
P_{n}^{(p, q)}(x)=\frac{(-1)^{n}(1+x)^{n}}{n ! 2^{n}} M_{n}^{(q, p+2 n+1)}\left(\frac{1-x}{1+x}\right) .
$$

By replacing (1.9) in (1.6), we can here obtain a new integral relation for the Jacobi polynomials straightforwardly:

$$
\int_{-1}^{1}(1-x)^{\alpha}(1+x)^{\beta-1}\left(P_{n}^{(\alpha, \beta)}(x)\right)^{2} d x=\frac{2^{\alpha+\beta}}{\beta} \frac{\Gamma(n+\alpha+1) \Gamma(n+\beta+1)}{\Gamma(n+1) \Gamma(n+\alpha+\beta+1)} .
$$

The second finite sequence of sextuplet classes, extracted from the main equation (1.1), is the polynomial set $\left\{N_{n}^{(p)}(x)\right\}_{n=0}^{n=N}$ that is finitely orthogonal with respect to the weight function $W_{2}(x, p)=x^{-p} e^{-1 / x}$ on $[0, \infty)$ if and only if $p>2 N+1[13$, Section 3].

These polynomials take the explicit form

$$
N_{n}^{(p)}(x)=(-1)^{n} \sum_{k=0}^{n} k !\left(\begin{array}{c}
p-(n+1) \\
k
\end{array}\right)\left(\begin{array}{c}
n \\
n-k
\end{array}\right)(-x)^{k}
$$

that satisfies the orthogonality relation

$$
\int_{0}^{\infty} x^{-p} e^{-1 / x} N_{n}^{(p)}(x) N_{m}^{(p)}(x) d x=\frac{n !(p-n-1) !}{p-2 n-1} \delta_{n, m} \Longleftrightarrow m, n=0,1,2, \ldots, N<\frac{p-1}{2} .
$$


As was seen in the fifth row of Table 1.1, these polynomials have a direct relation with the generalized Bessel polynomials $[16,24]$, so that their differential equation is a special case of $B_{n}^{(\alpha, \beta)}(x)$. Of course, it is necessary to point out that the orthogonality relation of the generalized Bessel polynomials has been showed on "unique circle" by Krall and Frink $[12]$ and not on $[0, \infty)$ finitely, as we have showed in [13] and relation (1.12).

Furthermore, we can here prove that the Bessel polynomials have a direct relation with the Laguerre polynomials too, because if the generalized Bessel equation

$$
x^{2} y^{\prime \prime}(x)+\left(r_{1} x+r_{2}\right) y^{\prime}(x)+r_{3} y(x)=0, \quad r_{2} \neq 0,
$$

is considered, then by considering the variable $y(x)=x^{r} F(1 / x)$ we have $F(x)=x^{r} y(1 / x)$, therefore (1.13) becomes

$$
x^{2} F^{\prime \prime}(x)+x\left(-r_{2} x+2-r_{1}-2 r\right) F^{\prime}(x)+\left(r r_{2} x+r^{2}+\left(r_{1}-1\right) r+r_{3}\right) F(x)=0
$$

Now if in this equation we assume that

$$
r^{2}+\left(r_{1}-1\right) r+r_{3}=0 \Longrightarrow r=\frac{1-r_{1} \pm \sqrt{\left(r_{1}-1\right)^{2}-4 r_{3}}}{2}
$$

then (1.14) changes to

$$
x F^{\prime \prime}(x)+\left(1 \mp \sqrt{\left(r_{1}-1\right)^{2}-4 r_{3}}-r_{2} x\right) F^{\prime}(x)+r_{2} \frac{1-r_{1} \pm \sqrt{\left(r_{1}-1\right)^{2}-4 r_{3}}}{2} F(x)=0 .
$$

But on the other hand, it is known that the solution of

$$
x g^{\prime \prime}(x)+(c-b x) g^{\prime}(x)-\operatorname{bag}(x)=0
$$

can be indicated by the confluent hypergeometric functions [16]

$$
g(x)={ }_{1} F_{1}(a, c ; b x)=\sum_{k=0}^{\infty} \frac{(a)_{k}}{(c)_{k}} \frac{(b x)^{k}}{k !} .
$$

Hence, by comparing (1.16) and (1.17), one can conclude that

$$
y(x)=x^{\left(1-r_{1} \pm \sqrt{\left(r_{1}-1\right)^{2}-4 r_{3}}\right) / 2}{ }_{1} F_{1}\left(\frac{r_{1}-1 \mp \sqrt{\left(r_{1}-1\right)^{2}-4 r_{3}}}{2}, 1 \mp \sqrt{\left(r_{1}-1\right)^{2}-4 r_{3}} ; \frac{r_{2}}{x}\right)
$$


satisfy (1.13) if and only if $r_{2} \neq 0$. So, the Bessel polynomials can be represented in terms of the Laguerre polynomials exactly, that is,

$$
\bar{B}_{n}^{(p)}(x)=\frac{(-1)^{n} n !(p+n) !}{(p+2 n) !} x^{n} L_{n}^{(-p-2 n-1)}\left(\frac{2}{x}\right),
$$

where $\bar{B}_{n}^{(\alpha)}(x)$ is the Bessel monic polynomials with the components $a=1, b=0, c=0$, $d=\alpha+2, e=2$ in the main equation (1.1). Accordingly, $N_{n}^{(p)}(x)$ can be represented by the Laguerre polynomials too, so that we have

$$
N_{n}^{(p)}(x)=n ! x^{n} L_{n}^{(p-2 n-1)}\left(\frac{1}{x}\right)
$$

This relation is useful to generate a new definite integral relation for the Laguerre polynomials, so that replacing (1.21) in (1.12) yields

$$
\int_{0}^{\infty} x^{\alpha-1} e^{-x}\left(L_{n}^{(\alpha)}(x)\right)^{2} d x=\frac{1}{\alpha} \frac{(n+\alpha) !}{n !} .
$$

Finally, the third finite orthogonal class, extracted from (1.1), is the polynomials set $\left\{J_{n}^{(p, q)}(x ; a, b, c, d)\right\}_{n=0}^{n=N}$ that is finitely orthogonal with respect to the weight function $W_{3}^{(p, q)}(x ; a, b, c, d)=\left((a x+b)^{2}+(c x+d)^{2}\right)^{-p} \exp (q \operatorname{Arctg}((a x+b) /(c x+d)))$ on $(-\infty, \infty)$ if and only if $q \in \mathbb{R}, a d-b c>0$ and $P>N+1 / 2$. Because of a special importance, this class was expressed in [14] separately and showed that the Rodrigues representation of this class

$$
\begin{aligned}
& J_{n}^{(p, q)}(x ; a, b, c, d)=(-1)^{n}\left((a x+b)^{2}+(c x+d)^{2}\right)^{p} \exp \left(-q \operatorname{Arctg} \frac{a x+b}{c x+d}\right) \\
& \times \frac{d^{n}\left(\left((a x+b)^{2}+(c x+d)^{2}\right)^{n-p} \exp (q \operatorname{Arctg}((a x+b) /(c x+d)))\right)}{d x^{n}}, \\
& \text { s.t. det }\left[\begin{array}{ll}
a & b \\
c & d
\end{array}\right]=a d-b c>0
\end{aligned}
$$

has the explicit form

$$
\begin{aligned}
J_{n}^{(p, q)}(x ; a, b, c, d)= & (-1)^{n}((a b+c d)+i(a d-b c))^{n}(n+1-2 p)_{n} \\
& \times \sum_{k=0}^{k=n}\left(\begin{array}{l}
n \\
k
\end{array}\right)\left(\frac{a^{2}+c^{2}}{(a b+c d)+i(a d-b c)}\right)^{k} \\
& \quad \times{ }_{2} F_{1}\left(\begin{array}{cc}
k-n & \left.p-n-\frac{i q}{2} \mid \frac{2(a d-b c)}{(a d-b c)-i(a b+c d)}\right) x^{k} \\
2 p-2 n
\end{array}\right.
\end{aligned}
$$


that satisfies the orthogonality relation

$$
\begin{gathered}
\int_{-\infty}^{\infty}\left((a x+b)^{2}+(c x+d)^{2}\right)^{-p} \exp \left(q \operatorname{Arctg} \frac{a x+b}{c x+d}\right) J_{n}^{(p, q)}(x ; a, b, c, d) J_{m}^{(p, q)}(x ; a, b, c, d) d x \\
=\left(\frac{n !\left(a^{2}+c^{2}\right)^{n} \Gamma(2 p-n)}{(a d-b c)^{2 p-2 n-1} \Gamma(2 p-2 n)} \int_{-\pi / 2}^{\pi / 2}(a \operatorname{Cos} \theta-c \operatorname{Sin} \theta)^{2 p-2 n-2} e^{q \theta} d \theta\right) \delta_{n, m}
\end{gathered}
$$

if and only if $m, n=0,1,2, \ldots, N<p-1 / 2$ and $a d-b c>0, q \in \mathbb{R}$.

In [13, Section 2], we also introduced a special case of $J_{n}^{(p, q)}(x ; a, b, c, d)$ for $q=0$ and called it as $I_{n}^{(p)}(x)$. Moreover, we proved that the finite subclass $\left\{I_{n}^{(p)}(x)\right\}_{n=0}^{n=N}=$ $\left\{n ! \sum_{k=0}^{[n / 2]}(-1)^{k}\left(\begin{array}{c}p-1 \\ n-k\end{array}\right)\left(\begin{array}{c}n-k \\ k\end{array}\right)(2 x)^{n-2 k}\right\}_{n=0}^{n=N}$ is orthogonal with respect to the weight function $W_{4}(x, p)=\left(1+x^{2}\right)^{-(p-1 / 2)}$ on $(-\infty, \infty)$ if and only if $p>N+1$ and the orthogonality relation

$$
\begin{aligned}
\int_{-\infty}^{\infty} & \left(1+x^{2}\right)^{-(p-1 / 2)} I_{n}^{(p)}(x) I_{m}^{(p)}(x) d x \\
& =\frac{n ! 2^{2 n-1} \sqrt{\pi} \Gamma^{2}(p) \Gamma(2 p-2 n)}{(p-n-1) \Gamma(p-n) \Gamma(p-n+1 / 2) \Gamma(2 p-n-1)} \delta_{n, m}
\end{aligned}
$$

holds if and only if $m, n=0,1,2, \ldots, N<p-1$.

But one of the applications of the finite classes of orthogonal polynomials, which can be important, is to approximate the functions finitely. In other words, under the Dirichlet conditions, one can consider any arbitrary precision degree $n=N$ for approximating the function $f(x)$. For instance, we consider the finite set

$$
A=\left\{J_{n}^{(4,1)}(x ; 1,0,0,1)\right\}_{n=0}^{n=3}=\left\{1,6 x-1,20 x^{2}-10 x-3,24 x^{3}-36 x^{2}-12 x+7\right\}
$$

which is orthogonal with respect to the weight $W_{3}^{(4,1)}(x ; 1,0,0,1)=\left(1+x^{2}\right)^{-4} \exp (\operatorname{Arctg} x)$ on $(-\infty, \infty)$ and satisfies

$$
\int_{-\infty}^{\infty} \frac{\exp (\operatorname{Arctg} x)}{\left(1+x^{2}\right)^{4}} J_{n}^{(4,1)}(x) J_{m}^{(4,1)}(x) d x=\frac{n !(7-n) !(2 S h \pi / 2)}{(7-2 n) \prod_{k=0}^{3-n}\left(1+(6-2 n-2 k)^{2}\right)} \delta_{n, m}, \quad m, n \leq 3 .
$$

This relation is valid for every values $m$ and $n$ less than 4 .

Hence, by noting the mentioned orthogonality relation and considering the members of set $A$, one can obtain a third-degree polynomial approximation for $f(x)$ as

$$
f(x) \cong c_{0}+c_{1}(6 x-1)+c_{2}\left(20 x^{2}-10 x-3\right)+c_{3}\left(24 x^{3}-36 x^{2}-12 x+7\right)
$$


which yields

$$
\begin{aligned}
(288 \operatorname{Sh} & \left.\frac{\pi}{2}\right) f(x) \\
\cong & \left(629 \int_{-\infty}^{\infty}\left(1+x^{2}\right)^{-4} e^{\operatorname{Arctg} x} f(x) d x\right) \\
& +\left(85 \int_{-\infty}^{\infty}\left(1+x^{2}\right)^{-4} e^{\operatorname{Arctg} x}(6 x-1) f(x) d x\right)(6 x-1) \\
& +\left(9 \int_{-\infty}^{\infty}\left(1+x^{2}\right)^{-4} e^{\operatorname{Arctg} x}\left(20 x^{2}-10 x-3\right) f(x) d x\right)\left(20 x^{2}-10 x-3\right) \\
& +\left(\int_{-\infty}^{\infty}\left(1+x^{2}\right)^{-4} e^{\operatorname{Arctg} x}\left(24 x^{3}-36 x^{2}-12 x+7\right) f(x) d x\right)\left(24 x^{3}-36 x^{2}-12 x+7\right) .
\end{aligned}
$$

It is well known that this approximation is accurate for any arbitrary third-degree polynomial function, but if one wants to consider a precise expansion for an arbitrary polynomial function of degree $m$, then, for example, we can refer to the relation

$$
x^{m}=\sum_{n=0}^{n=m} \frac{(p-(2 n+1))(p-(n+m+2)) !(q+m) !}{(p-(n+1)) !(q+n) !}\left(\begin{array}{c}
m \\
n
\end{array}\right) M_{n}^{(p, q)}(x)
$$

which is a basis space for any arbitrary polynomial function of degree $m$ (see [13]).

\section{Six rational classical orthogonal polynomials}

Again, we consider the differential equation (1.1). Clearly one of the main solution of this equation is a polynomial-like $P_{n}(x ; a, b, c, d, e)$. In [11], we have proved that the monic polynomial solution of (1.1) takes the form

$$
\begin{aligned}
\bar{P}_{n}\left(\begin{array}{lll}
d & & e \\
a & b & c
\end{array} \mid x\right)= & \sum_{k=0}^{k=n}\left(\begin{array}{l}
n \\
k
\end{array}\right)\left(\frac{2 a}{b+\sqrt{b^{2}-4 a c}}\right)^{k-n} \\
& \times{ }_{2} F_{1}\left(\begin{array}{c}
\left.k-n \frac{2 a e-b d}{2 a \sqrt{b^{2}-4 a c}}+1-n-\frac{d}{2 a} \mid \frac{2 \sqrt{b^{2}-4 a c}}{b+\sqrt{b^{2}-4 a c}}\right) x^{k} \\
2-2 n-\frac{d}{a}
\end{array}\right.
\end{aligned}
$$

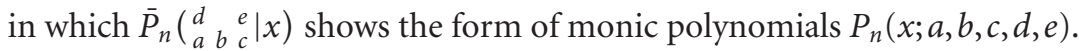

Now, if the Mobius transform $x=p z^{-1}+q, p \neq 0, q \in \mathbb{R}$ is applied for (1.1), then it eventually changes to the general form

$$
x^{2}\left(l_{2} x^{2}+l_{1} x+l_{0}\right) y^{\prime \prime}+x\left(2 l_{2} x^{2}+l_{3} x+l_{4}\right) y^{\prime}-n\left((n+1) l_{0}-l_{4}\right) y=0,
$$

where $l_{4}, l_{3}, l_{2}, l_{1}, l_{0}$ are real parameters and $n$ is a positive integer number. 
Obviously one of the main solution of this equation is a rational (negative power)

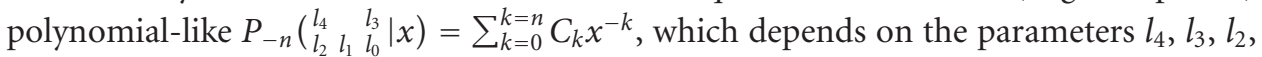
$l_{1}, l_{0}$, respectively. But according to the Sturm-Liouville theorem, one can find the weight function, corresponding to the differential equation (2.2), as the generic form

$$
W\left(\begin{array}{lll}
l_{4} & & l_{3} \\
l_{2} & l_{1} & l_{0}
\end{array} \mid x\right)=\exp \left(\int \frac{-2 l_{2} x^{2}+\left(l_{3}-3 l_{1}\right) x+\left(l_{4}-2 l_{0}\right)}{x\left(l_{2} x^{2}+l_{1} x+l_{0}\right)} d x\right) .
$$

If we assume that $[L, U]$ is the orthogonality interval, then

$$
\int_{L}^{U} W\left(\begin{array}{lll}
l_{4} & & l_{3} \\
l_{2} & l_{1} & l_{0}
\end{array} \mid x\right) P_{-n}\left(\begin{array}{lll}
l_{4} & & l_{3} \\
l_{2} & l_{1} & l_{0}
\end{array} \mid x\right) P_{-m}\left(\begin{array}{lll}
l_{4} & & l_{3} \\
l_{2} & l_{1} & l_{0}
\end{array} \mid x\right) d x=A_{n} \delta_{n, m}
$$

is the orthogonality relation of rational classical orthogonal polynomials, in which

$$
A_{n}=\int_{L}^{U} W\left(\begin{array}{lll}
l_{4} & & l_{3} \\
l_{2} & l_{1} & l_{0}
\end{array} \mid x\right)\left(P_{-n}\left(\begin{array}{lll}
l_{4} & & l_{3} \\
l_{2} & l_{1} & l_{0}
\end{array} \mid x\right)\right)^{2} d x
$$

is the norm square value. There are six cases, corresponding to the main differential equation (2.2), that are orthogonal for some specific values of $l_{4}, l_{3}, l_{2}, l_{1}, l_{0}$.

As was mentioned, the connection between (1.1) and (2.2) is the Mobius transform $x=p z^{-1}+q$ for different values of $p$ and $q$. So, as an example, we here consider the Mobius transform for Jacobi rational polynomials $P_{-n}^{(\alpha, \beta)}(x)$. If $p=-2, q=1$, is considered, then we define

$$
P_{-n}^{(\alpha, \beta)}(x)=P_{n}^{(\alpha-\beta-2, \beta)}\left(-2 x^{-1}+1\right)=\sum_{k=0}^{k=n}(-1)^{k}\left(\begin{array}{c}
n+\alpha-2+k \\
k
\end{array}\right)\left(\begin{array}{c}
n+\alpha-2+\beta \\
n-k
\end{array}\right) x^{-k}
$$

For instance, $n=0,1,2$ in (2.6) gives

$$
\begin{gathered}
P_{0}^{(\alpha, \beta)}(x)=1, \\
P_{-1}^{(\alpha, \beta)}(x)=-\alpha x^{-1}+(\alpha-\beta-1), \\
P_{-2}^{(\alpha, \beta)}(x)=\frac{1}{2}(\alpha+2)(\alpha+1) x^{-2}-(\alpha+1)(\alpha-\beta) x^{-1}+\frac{1}{2}(\alpha-\beta)(\alpha-\beta-1) .
\end{gathered}
$$

Since the orthogonality relation of Jacobi polynomials is known, the orthogonality relation of $P_{-n}^{(\alpha, \beta)}(x)$ will be

$$
\begin{aligned}
\int_{1}^{\infty} x^{-\alpha}(x-1)^{\beta} P_{-n}^{(\alpha, \beta)}(x) P_{-m}^{(\alpha, \beta)}(x) d x \\
\quad=\frac{(n+\alpha-\beta-2) !(n+\beta) !}{(2 n+\alpha-1) n !(n+\alpha-2) !} \delta_{n, m} \Longleftrightarrow \alpha>0, \quad \beta>-1 .
\end{aligned}
$$


The differential equation of $y=P_{-n}^{(\alpha, \beta)}(x)$ is a special case of the main equation (2.2) for $l_{4}=\alpha-2, l_{3}=\beta-\alpha+3, l_{2}=0, l_{1}=1, l_{0}=-1$, that is, we have

$$
x^{2}(x-1) y^{\prime \prime}+x((\beta-\alpha+3) x+\alpha-2) y^{\prime}+n(n+\alpha+1) y=0
$$

Easily one can apply the Mobius transforms for five other classes of classical orthogonal polynomials. However, note that all differential equations of these transforms must be special cases of the main equation (2.2). For instance, the Laguerre rational orthogonal polynomials $L_{-n}^{(\alpha)}(x)=L_{n}^{(\alpha)}\left(x^{-1}-1\right)$ satisfy the equation

$$
x^{3}(1-x) y^{\prime \prime}-x\left(2 x^{2}+\alpha x-1\right) y^{\prime}+n y=0
$$

which is clearly a special case of (2.2), or the equation

$$
x^{4} y^{\prime \prime}+2 x\left(x^{2}+\alpha \beta x+\alpha^{2}\right) y^{\prime}+2 n \alpha^{2} y=0
$$

is the differential equation of the Hermite rational orthogonal polynomials, defined by $H_{-n}(x)=H_{n}\left(\alpha x^{-1}+\beta\right)$.

\section{Evaluation of inverse Laplace transform using rational classical orthogonal polynomials}

It is known that the Laplace transformation provides a powerful method for analyzing linear systems, however, many physical problems lead to Laplace transforms whose inverses are not readily expressed in terms of tabulated functions. Hence, so far extensive researches have been done on evaluating this matter and its applications.

For instance, Chandran [3] has recently computed inverse Laplace transforms of a class of nonrational fractional functions, and Evans and Chung [7] have obtained Laplace transform inversions by applying optimal contours in the complex plane, see also [1]. Also, a classroom note regarding Fourier method for inversion of Laplace transform has been stated by Iqbal in [9]. The problem of inverse two-sided Laplace transform for probability density functions is another topic that has been stated by Tagliani in 1998 [25].

On the other hand, the problem of numerical inversion of Laplace transform has been studied by several authors. For example, Cunha and Viloche [5] have presented an iterative method for the Numerical inversion of Laplace transform, and Dong [6] has introduced a regularization method for this purpose. In [4], Crump has used Fourier series approximation (see also [8] in this regard) while Miller and Guy in [15] have used Jacobi polynomials, and Sidi [23] has applied a window function for Laplace transform inversion. Finally, Piessens' works $[19,20]$ are a good bibliography that one can refer to it for the Laplace transform numerical inversion.

But in cases where the inverse Laplace transform is required for many values of the independent variable, it is convenient to obtain the inverse as a series expansion in terms of a set of linearly independent functions. Procedure based on this idea can be calculated by solving a system of equations that can be reduced to a triangular system if one chooses to use "orthogonal polynomials." Such a method, using orthogonal polynomials, gives an approximate evaluation of the inversion integral using "Gauss quadrature" in 


\section{Orthogonal polynomials and inverse Laplace transforms}

the complex plane $[17,18,21,22]$. Of course, the chief disadvantage of this method is the necessity of finding all roots, real and complex, of a polynomial of high degree, and of the calculation of a set of complex Christoffel numbers [26, page 419]. So, here we would like to insist that the orthogonal polynomials with negative powers are a suitable tool to compute the inverse Laplace transform without any problem in finding the roots of orthogonal polynomials. To reach this goal, we refer to the orthogonality properties introduced in Section 2 and give three basic expansions, as a sample, for Laplace transform inversion.

\subsection{Inverse Laplace transform using the Jacobi rational orthogonal polynomials} $P_{-n}^{(\alpha, \beta)}(x)$. We consider the Laplace transform along with its inverse

$$
F(s)=L[f(x)]=\int_{0}^{\infty} e^{-s x} f(x) d x \Longleftrightarrow f(x)=L^{-1}[F(s)]=\frac{1}{2 \pi i} \int_{\lambda-i \infty}^{\lambda+i \infty} e^{s x} F(s) d s \quad \forall s>0 .
$$

We can find a solution for the integral equation (3.1) provided that $F(s)$ is known and expandable. By noting the orthogonality relation (2.8), first it is concluded that

$$
\int_{0}^{\infty}(t+1)^{-\alpha} t^{\beta} P_{-n}^{(\alpha, \beta)}(t+1) P_{-m}^{(\alpha, \beta)}(t+1) d t=\frac{(n+\alpha-\beta-2) !(n+\beta) !}{(2 n+\alpha-1) n !(n+\alpha-2) !} \delta_{n, m} .
$$

Now suppose $F(s)$ satisfies the Dirichlet conditions and

$$
F(s)=\sum_{n=0}^{\infty} C_{n} P_{-n}^{(\alpha, \beta)}(s+1)=\sum_{n=0}^{\infty} \frac{A_{n}}{(s+1)^{n}} .
$$

Applying (3.2) to (3.3), the coefficients $C_{n}$ will be

$$
C_{n}=\frac{(2 n+\alpha-1) n !(n+\alpha-2) !}{(n+\alpha-\beta-2) !(n+\beta) !} \int_{0}^{\infty}(s+1)^{-\alpha} s^{\beta} P_{-n}^{(\alpha, \beta)}(s+1) F(s) d s
$$

On the other hand, taking inverse transform from (3.3) gives

$$
f(x)=L^{-1}[F(s)]=\sum_{n=0}^{\infty} C_{n} L^{-1}\left[P_{-n}^{(\alpha, \beta)}(s+1)\right] .
$$

But according to the definition (2.6), we have

$$
\begin{aligned}
L^{-1}\left[P_{-n}^{(\alpha, \beta)}(s+1)\right] & =\sum_{k=0}^{k=n}(-1)^{k}\left(\begin{array}{c}
n+\alpha-2+k \\
k
\end{array}\right)\left(\begin{array}{c}
n+\alpha-2-\beta \\
n-k
\end{array}\right) L^{-1}\left[(s+1)^{-k}\right] \\
& =\left(\begin{array}{c}
n+\alpha-2-\beta \\
\alpha-2-\beta
\end{array}\right) \delta(x)-e^{-x} \sum_{k=1}^{k=n}\left(\begin{array}{c}
n+\alpha-2+k \\
k
\end{array}\right)\left(\begin{array}{c}
n+\alpha-2-\beta \\
n-k
\end{array}\right) \frac{(-x)^{k-1}}{(k-1) !}
\end{aligned}
$$


in which $\delta(x)=\operatorname{Lim}_{\varepsilon \rightarrow 0} f_{\varepsilon}(x)$ s.t.

$$
f_{\varepsilon}(x)= \begin{cases}\frac{1}{\varepsilon} & 0 \leq x \leq \varepsilon \\ 0 & x>\varepsilon\end{cases}
$$

is the Dirac function. So the special series

$$
\begin{aligned}
f(x)= & \left(\frac{(\alpha-1) !}{(\alpha-\beta-2) ! \beta !} \int_{0}^{\infty}(s+1)^{-\alpha} s^{\beta} F(s) d s\right) \delta(x) \\
& +\sum_{n=1}^{\infty}\left(\frac{(2 n+\alpha-1) n !(n+\alpha-2) !}{(n+\alpha-\beta-2) !(n+\beta) !} \int_{0}^{\infty}(s+1)^{-\alpha} s^{\beta} P_{-n}^{(\alpha, \beta)}(s+1) F(s) d s\right) \\
& \quad \times\left(\left(\begin{array}{c}
n+\alpha-2-\beta \\
\alpha-2-\beta
\end{array}\right) \delta(x)-e^{-x} \sum_{k=1}^{k=n}\left(\begin{array}{c}
n+\alpha-2+k \\
k
\end{array}\right)\left(\begin{array}{c}
n+\alpha-2-\beta \\
n-k
\end{array}\right) \frac{(-x)^{k-1}}{(k-1) !}\right)
\end{aligned}
$$

is an expansion solution for (3.1). It should be noted that this solution is valid if and only if its definite integrals are convergent and the function $F(s)$ is expandable in (3.3) under the Dirichlet conditions. The function $(s+1)^{-\alpha} s^{\beta}$ is in fact a weight distribution for computation of the definite integrals of $(3.8)$ on $[0, \infty)$. Hence, if the weight distribution changes, we will reach another expansion to compute the inverse Laplace transform. Next section will show this matter.

3.2. Inverse Laplace transform using the Laguerre rational orthogonal polynomials $L_{-n}^{(\alpha)}(x)$. First, we define the sequence

$$
L_{-n}^{\alpha}(s)=L_{n}^{(\alpha)}\left(\frac{1}{s}\right)=\sum_{k=0}^{k=n} \frac{(-1)^{k}}{k !}\left(\begin{array}{l}
n+\alpha \\
n-k
\end{array}\right) s^{-k}
$$

that satisfies the orthogonality relation

$$
\int_{0}^{\infty} s^{-(\alpha+2)} e^{-1 / s} L_{-n}^{(\alpha)}(s) L_{-m}^{(\alpha)}(s) d s=\frac{(n+\alpha) !}{n !} \delta_{n, m} .
$$

Now if the expansion

$$
F(s)=\sum_{n=0}^{\infty} C_{n} L_{-n}^{(\alpha)}(s)=\sum_{n=0}^{\infty} \frac{A_{n}}{s^{n}}
$$

is considered, then by applying (3.10) to (3.11), the coefficients $C_{n}$ will be

$$
C_{n}=\frac{n !}{(n+\alpha) !} \int_{0}^{\infty} s^{-(\alpha+2)} e^{-1 / s} L_{-n}^{(\alpha)}(s) F(s) d s .
$$


Therefore,

$$
f(x)=L^{-1}[F(s)]=\sum_{n=0}^{\infty} C_{n} L^{-1}\left[L_{-n}^{(\alpha)}(s)\right]
$$

But on the other hand, we have

$$
L^{-1}\left[L_{-n}^{(\alpha)}(s)\right]=\sum_{k=0}^{k=n} \frac{(-1)^{k}}{k !}\left(\begin{array}{l}
n+\alpha \\
n-k
\end{array}\right) L^{-1}\left[\frac{1}{s^{k}}\right]
$$

So, the special series

$$
\begin{aligned}
f(x)= & \left(\frac{1}{\alpha !} \int_{0}^{\infty} s^{-(\alpha+2)} e^{-1 / s} F(s) d s\right) \delta(x) \\
& +\sum_{n=1}^{\infty}\left(\frac{n !}{(n+\alpha) !} \int_{0}^{\infty} s^{-(\alpha+2)} e^{-1 / s} L_{-n}^{(\alpha)}(s) F(s) d s\right)\left(\left(\begin{array}{c}
n+\alpha \\
n
\end{array}\right) \delta(x)-\sum_{k=1}^{k=n}\left(\begin{array}{c}
n+\alpha \\
n-k
\end{array}\right) \frac{(-x)^{k-1}}{k !(k-1) !}\right)
\end{aligned}
$$

would be a solution for Laplace transform inversion. Again, we mention that (3.15) is valid only if its definite integrals are convergent and the function $F(s)$ is expandable under the Dirichlet conditions. In relation (3.15), the function $s^{-(\alpha+2)} e^{-1 / s}$ plays the weight distribution role on $[0, \infty)$.

By noting Section 1, finite rational classical orthogonal polynomials can also be applied for approximate computation of inverse Laplace transform, because one can expand the function $F(s)$ by them finitely, and just some limit conditions impose on their parameters. For example, if we consider the sequence

$$
M_{-n}^{(p, q)}(s)=M_{n}^{(p+2, q)}\left(\frac{1}{s}\right)=(-1)^{n} n ! \sum_{k=0}^{k=n}(-1)^{k}\left(\begin{array}{c}
p+1-n \\
k
\end{array}\right)\left(\begin{array}{c}
q+n \\
n-k
\end{array}\right) s^{-k}
$$

that satisfies the orthogonality relation

$$
\int_{0}^{\infty} \frac{s^{p}}{(1+s)^{p+q+2}} M_{-n}^{(p, q)}(s) M_{-m}^{(p, q)}(s) d s=\frac{n !(p+1-n) !(q+n) !}{(p+1-2 n)(p+q+1-n) !} \delta_{n, m},
$$

provided that $q>-1, p>2 N-1, N=\operatorname{Max}\{m, n\}$, then by taking the approximation

$$
F(s) \cong \sum_{n=0}^{N} C_{n} M_{-n}^{(p, q)}(s)=\sum_{n=0}^{N} \frac{A_{n}}{s^{n}}, \quad N<\frac{p+1}{2}
$$

and applying (3.17) to (3.18), we have

$$
C_{n}=\frac{(p+1-2 n)(p+q+1-n) !}{n !(p+1-n) !(q+n) !} \int_{0}^{\infty} \frac{s^{p}}{(1+s)^{p+q+2}} M_{-n}^{(p, q)}(s) F(s) d s .
$$


Therefore,

$$
f(x)=L^{-1}[F(s)] \cong \sum_{n=0}^{N} C_{n} L^{-1}\left[M_{-n}^{(p, q)}(s)\right]
$$

in which

$$
L^{-1}\left[M_{-n}^{(p, q)}(s)\right]=(-1)^{n} n ! \sum_{k=0}^{k=n}(-1)^{k}\left(\begin{array}{c}
p+1-n \\
k
\end{array}\right)\left(\begin{array}{c}
q+n \\
n-k
\end{array}\right) L^{-1}\left[\frac{1}{s^{k}}\right] .
$$

Hence,

$$
\begin{aligned}
& f(x) \cong\left(\frac{(p+q+1) !}{p ! q !} \int_{0}^{\infty} \frac{s^{p}}{(1+s)^{p+q+2}} F(s) d s\right) \delta(x) \\
& +\sum_{n=1}^{N}\left(\frac{(p+1-2 n)(p+q+1-n) !}{n !(p+1-n) !(q+n) !} \int_{0}^{\infty} \frac{s^{p}}{(1+s)^{p+q+2}} M_{-n}^{(p, q)}(s) F(s) d s\right) \\
& \times\left(\left(\begin{array}{c}
q+n \\
n
\end{array}\right) \delta(x)-(-1)^{n} n ! \sum_{k=1}^{k=n}\left(\begin{array}{c}
p+1-n \\
k
\end{array}\right)\left(\begin{array}{c}
q+n \\
n-k
\end{array}\right) \frac{(-x)^{k-1}}{(k-1) !}\right)
\end{aligned}
$$

is an approximate solution for the equation $L[f(x)]=F(s)$. We mention that one can also propound the inversion problem for the negative power polynomials $N_{-n}^{(p)}(x), H_{-n}(x)$, and $J_{-n}^{(p, q)}(x ; a, b, c, d)$ similarly. Here is a good position to propound two practical examples for Section 3.

\section{Special examples of Section 3}

Example 4.1. We consider a special case of Jacobi rational polynomials for $\alpha=1, \beta=$ $-1 / 2$. This case is defined as

$$
P_{-n}^{(1,-1 / 2)}(x+1)=T_{n}\left(\frac{x-1}{x+1}\right)=\operatorname{Cos}\left(n \operatorname{Arccos} \frac{x-1}{x+1}\right)
$$

and called the Chebyshev rational polynomials of first kind [24]. Now by noting the expansion (3.8) suppose, for instance, $F(s)=1 /(1+s)$. This implies that the integrals of (3.8) are simplified as

$$
\begin{aligned}
& \int_{0}^{\infty} \frac{s^{-1 / 2}}{(1+s)^{2}} d s=\frac{\pi}{2}, \\
& \int_{0}^{\infty} \frac{s^{-1 / 2}}{(1+s)^{2}} P_{-n}^{(1,-1 / 2)}(s) d s=\sum_{k=0}^{k=n}\left(\begin{array}{c}
n+k-1 \\
k
\end{array}\right)\left(\begin{array}{c}
n-\frac{1}{2} \\
n-k
\end{array}\right) \int_{0}^{\infty} \frac{s^{-1 / 2}}{(1+s)^{k+2}} d s \\
&=\frac{\sqrt{\pi} \Gamma(n+1 / 2)}{2 \Gamma(n)} \sum_{k=0}^{k=n} \frac{(-1)^{k}(n+k-1) !(2 k+1)}{k !(n-k) !(k+1) !} .
\end{aligned}
$$


228 Orthogonal polynomials and inverse Laplace transforms

Therefore, the inverse Laplace transform for the given $F(s)$ takes the form

$$
\begin{aligned}
L^{-1}\left(\frac{1}{1+s}\right)=e^{-x}= & \frac{1}{2} \delta(x) \\
+ & \sum_{n=1}^{\infty}\left(\frac{n \sqrt{\pi} \Gamma(n+1)}{2 \Gamma(n)} \sum_{i=0}^{i=n} \frac{(-1)^{i}(n+i-1) !(2 i+1)}{i !(n-i) !(i+1) !}\right) \\
& \times\left(\frac{\Gamma(n+1 / 2)}{\sqrt{\pi} n !} \delta(x)-e^{-x} \sum_{k=1}^{k=n}\left(\begin{array}{c}
n+k-1 \\
k
\end{array}\right)\left(\begin{array}{c}
n-\frac{1}{2} \\
n-k
\end{array}\right) \frac{(-x)^{k-1}}{(k-1) !}\right) .
\end{aligned}
$$

Clearly the above relation is valid if it is expanded.

Example 4.2. For this example, we use the analytical expansion (3.15), mentioned in Section 3.2, and consider the equation $L[f(x)]=\sqrt{\pi} / 2 s \sqrt{s}$. To derive the solution of this equation, first we have to evaluate the definite integrals of (3.15). Hence, we have

$$
\begin{aligned}
F(s) & =\frac{\sqrt{\pi}}{2 s \sqrt{s}} \Longrightarrow \int_{0}^{\infty} s^{-(\alpha+2)} e^{-1 / s} F(s) d s=\frac{\sqrt{\pi}}{2} \Gamma\left(\alpha+\frac{5}{2}\right), \\
\int_{0}^{\infty} s^{-(\alpha+2)} e^{-1 / s} L_{-n}^{(\alpha)}(s) F(s) d s & \\
& =\frac{\sqrt{\pi}}{2} \sum_{k=0}^{k=n} \frac{(-1)^{k}}{k !}\left(\begin{array}{c}
n+\alpha \\
n-k
\end{array}\right) \int_{0}^{\infty} s^{-(\alpha+7 / 2+k)} e^{-1 / s} d s \\
& =\frac{\sqrt{\pi} \Gamma(n+1+\alpha) \Gamma(\alpha+5 / 2)}{\Gamma(n+1) \Gamma(\alpha+1)}{ }_{2} F_{1}\left(\begin{array}{c}
-n \\
\alpha+\frac{5}{2}
\end{array} \mid 1\right) \\
& =\frac{3}{8} \frac{\Gamma(-n-3 / 2) \Gamma(\alpha+1+n) \Gamma(\alpha+5 / 2)}{\Gamma(n+1) \Gamma(\alpha+1-n)} .
\end{aligned}
$$

Note that for the latter integral, we have used the well-known Gauss identity [24], that is,

$$
{ }_{2} F_{1}\left(\begin{array}{cc|c}
a & b & 1 \\
c & 1
\end{array}\right)=\frac{\Gamma(c) \Gamma(c-a-b)}{\Gamma(c-a) \Gamma(c-b)} ; \quad c \neq 0,-1,-2, \ldots, c>a+b .
$$

Consequently the expansion (3.15) becomes

$$
\begin{aligned}
L^{-1}\left(\frac{\sqrt{\pi}}{2 s \sqrt{s}}\right)=\sqrt{x}= & \frac{\sqrt{\pi}}{2} \frac{\Gamma(\alpha+5 / 2)}{\Gamma(\alpha+1)} \delta(x) \\
+\sum_{n=1}^{\infty} & \left(\frac{3}{8} \frac{\Gamma(-n-3 / 2) \Gamma(\alpha+5 / 2)}{\Gamma(\alpha+1-n)}\right) \\
& \times\left(\left(\begin{array}{c}
n+\alpha \\
n
\end{array}\right) \delta(x)-\sum_{k=1}^{k=n}\left(\begin{array}{c}
n+\alpha \\
n-k
\end{array}\right) \frac{(-x)^{k-1}}{k !(k-1) !}\right) .
\end{aligned}
$$

This relation holds if and only if $\alpha>-1$ and $\alpha+1-n \notin \mathbb{Z}^{-}$. 


\section{Conclusion}

In this paper, first we had a survey on classical orthogonal polynomials, particularly on finite classes, and their corresponding rational orthogonal polynomials. Then we presented some integral relations for Jacobi, Laguerre, and Bessel orthogonal polynomials. Finally, in Section 3, by using the rational classical orthogonal polynomials, we applied a direct approach to compute the inverse Laplace transforms explicitly and presented three basic expansions as a sample. In this way, two practical examples were also given.

\section{References}

[1] D. D. Ang, J. Lund, and F. Stenger, Complex variable and regularization methods of inversion of the Laplace transform, Math. Comp. 53 (1989), no. 188, 589-608.

[2] S. Bochner, Uber Sturm-Liouvillesche Polynomsysteme, Math. Z. 29 (1929), 730-736 (German).

[3] P. Chandran, Inverse Laplace transforms of a class of non-rational fractional functions, Internat. J. Math. Ed. Sci. Tech. 32 (2001), no. 1, 136-140.

[4] K. S. Crump, Numerical inversion of Laplace transforms using a Fourier series approximation, J. Assoc. Comput. Mach. 23 (1976), no. 1, 89-96.

[5] C. Cunha and F. Viloche, An iterative method for the numerical inversion of Laplace transforms, Math. Comp. 64 (1995), no. 211, 1193-1198.

[6] C. W. Dong, A regularization method for the numerical inverse of the Laplace transform, SIAM J. Numer. Anal. 30 (1993), no. 3, 759-773.

[7] G. A. Evans and K. C. Chung, Laplace transform inversions using optimal contours in the complex plane, Int. J. Comput. Math. 73 (2000), no. 4, 531-543.

[8] C. Hwang, R. Y. Wu, and M. J. Lu, A technique for increasing the accuracy of the FFT-based method of numerical inversion of Laplace transforms, Comput. Math. Appl. 27 (1994), no. 7, 23-29.

[9] M. Iqbal, Fourier method for Laplace transform inversion, J. Appl. Math. Decis. Sci. 5 (2001), no. 3, 193-200.

[10] R. Koekoek and R. F. Swarttouw, The Askey-scheme of hypergeometric orthogonal polynomials and its q-analogue, Tech. Report 98-17, Faculty of Technical Mathematics and Informatics, Technical Universiteit Delft, Delft, 1998, http://aw.twi.tudelft.nl/ koekoek/askey/.

[11] W. Koepf and M. Masjed-Jamei, A generic polynomial solution for the differential equation of hypergeometric type and six sequences of classical orthogonal polynomials related to it, submitted to J. Comput. Appl. Math.

[12] H. L. Krall and O. Frink, A new class of orthogonal polynomials: The Bessel polynomials, Trans. Amer. Math. Soc. 65 (1949), 100-115.

[13] M. Masjed-Jamei, Three finite classes of hypergeometric orthogonal polynomials and their application in functions approximation, Integral Transforms Spec. Funct. 13 (2002), no. 2, 169191.

[14] Classical orthogonal polynomials with weight function $\left((a x+b)^{2}+(c x+\right.$ $\left.d)^{2}\right)^{-p} \exp (q \operatorname{Arctg}((a x+b) /(c x+d))), x \in(-\infty, \infty)$ and a generalization of $T$ and $F$ distributions, Integral Transforms Spec. Funct. 15 (2004), no. 2, 137-153.

[15] M. K. Miller and W. T. Guy, Numerical inversion of the Laplace transform by use of Jacobi polynomials, SIAM J. Numer. Anal. 3 (1966), no. 4, 624-635.

[16] A. F. Nikiforov and V. B. Uvarov, Special Functions of Mathematical Physics, Birkhäuser Verlag, Basel, 1988.

[17] R. Piessens, Gaussian quadrature formulas for the numerical integration of Bromwich's integral and the inversion of the Laplace transform, J. Engrg. Math. 5 (1971), no. 1, 1-9. 

, Gaussian quadrature formulas for Bromwich's integral, Comm. ACM 16 (1973), no. 8, $486-487$.

[19] A bibliography on numerical inversion of the Laplace transform and applications, J. Comput. Appl. Math. 1 (1975), no. 2, 115-128.

[20] R. Piessens and N. D. P. Dang, A bibliography on numerical inversion of the Laplace transform and applications: a supplement, J. Comput. Appl. Math. 2 (1976), no. 3, 225-228.

[21] H. E. Salzer, Orthogonal polynomials arising in the numerical evaluation of inverse Laplace transforms, Math. Tables Aids Comput. 9 (1955), no. 52, 164-177.

[22] J. Shohat, Laguerre polynomials and the Laplace transform, Duke Math. J. 6 (1940), 615-626.

[23] A. Sidi, Best rational function approximation to Laplace transform inversion using a window function, J. Comput. Appl. Math. 2 (1976), no. 3, 187-194.

[24] G. Szegö, Orthogonal Polynomials, 4th ed., American Mathematical Society, Rhode Island, 1975.

[25] A. Tagliani, Inverse two-sided Laplace transform for probability density functions, J. Comput. Appl. Math. 90 (1998), no. 2, 157-170.

[26] W. T. Weeks, Numerical inversion of Laplace transforms using Laguerre functions, J. Assoc. Comput. Mach. 13 (1966), no. 3, 419-429.

Mohammad Masjed-Jamei: Center of Research and Studies, Sanjesh Organization, Ministry of Science and Technology, Tehran, Iran

Current address: Department of Mathematics, K. N. Toosi University of Technology, P.O. Box 16315-1618, Tehran, Iran

E-mail address: mmjamei@aut.ac.ir

Mehdi Dehghan: Department of Applied Mathematics, Faculty of Mathematics and Computer Science, Amirkabir University of Technology, 424 Hafez Avenue, P.O. Box 15875-4413, Tehran, Iran

E-mail address: mdehghan@aut.ac.ir 


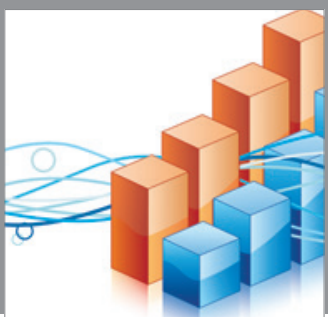

Advances in

Operations Research

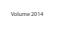

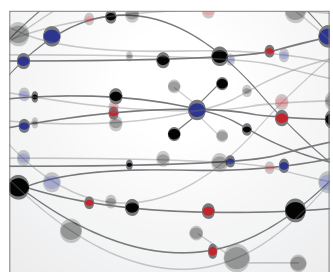

\section{The Scientific} World Journal
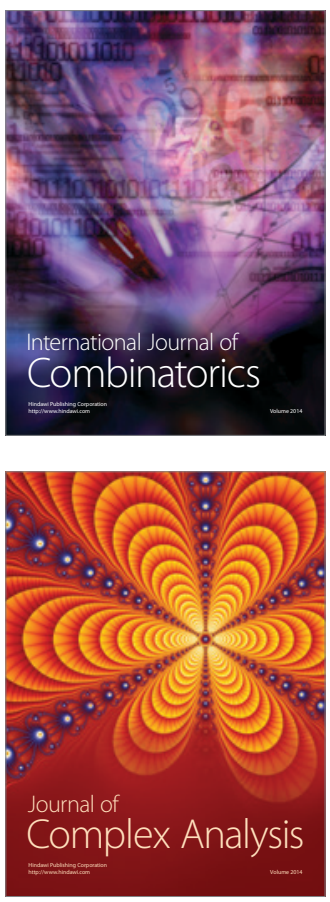

International Journal of

Mathematics and

Mathematical

Sciences
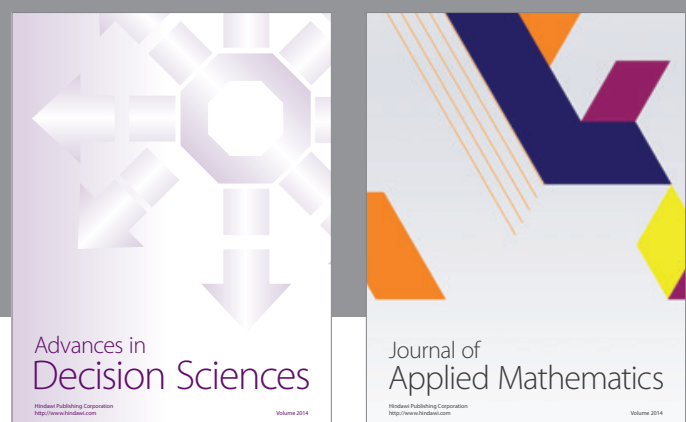

Journal of

Applied Mathematics


Submit your manuscripts at http://www.hindawi.com
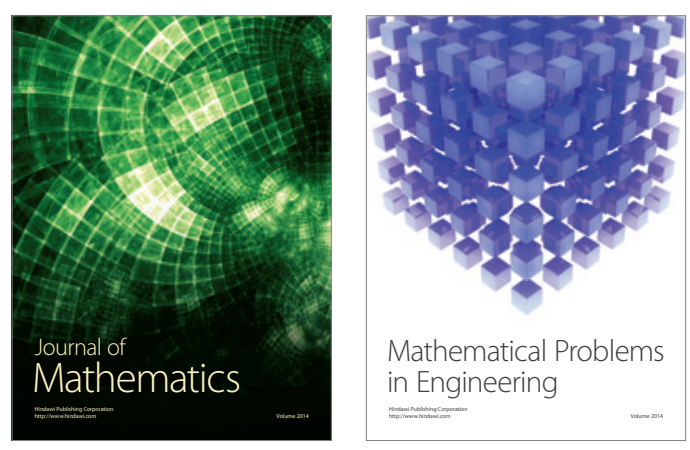

Mathematical Problems in Engineering
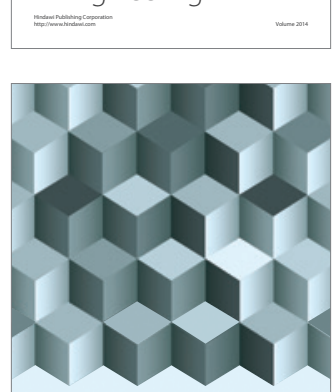

Journal of

Function Spaces
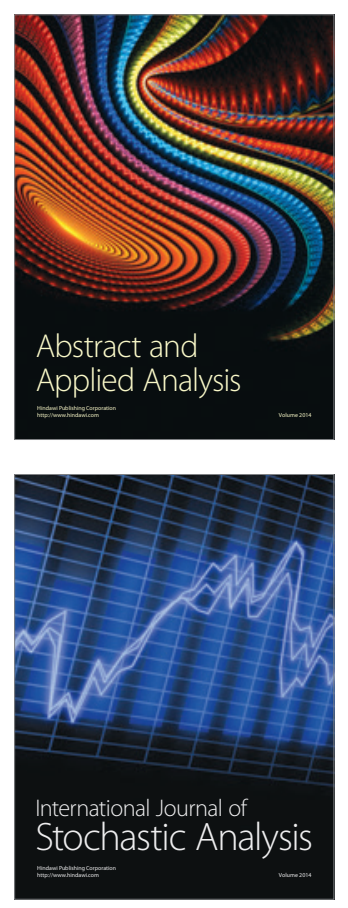

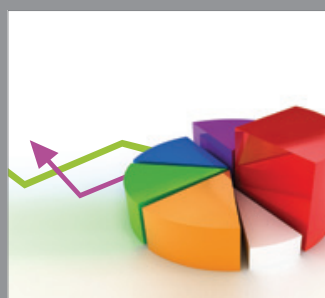

ournal of

Probability and Statistics

Promensencen
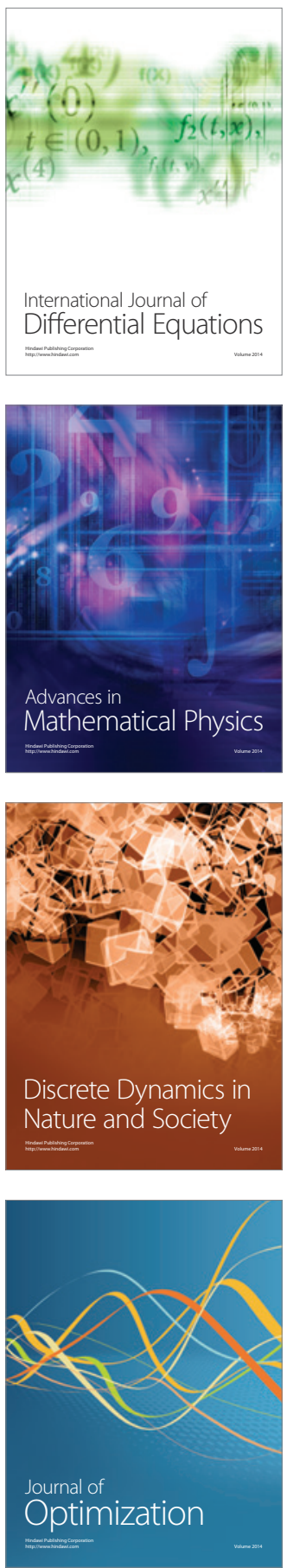